\title{
Post-Operative Intussusception in a Child after Pseudo Cyst of Pancreas Excision
}

\author{
Aditya Pratap Singh*, Arun Kumar Gupta, Dinesh Kumar Barolia, Maryem Ansari \\ Department of Pediatric Surgery, S.M.S. Medical College and Group of Hospitals, Jaipur, Rajasthan, India.
}

\begin{abstract}
Intussusception is commonly seen in pediatric age group. It is rarely present as a cause of post-operative obstruction. Ileus and adhesion band are the common causes of postoperative obstruction. The classical presentation of intussusception is frequently absent in post-operative intussusception, so high degree of suspicious is required for early diagnosis. Timely intervention can give excellent results, so we have to consider it as a differential diagnosis in post-operative obstruction in pediatric age group. We are presenting here a case of post-operative intussusception in one and half year old male child after excision of pseudo cyst of pancreas.
\end{abstract}

Keywords: Intussusception, Postoperative, Pseudo cyst, Pancreas, Obstruction, Excision, Child, Mesenteric Cyst.

\section{INTRODUCTION}

Bowel obstruction is common in pediatric age after abdominal surgery. Postoperative intussusception (POI) is rarely seen in pediatric age group as cause of bowel obstruction [1]. Intussusception causes bowel obstruction by the invagination of a part of bowel into another. POI usually has a short symptom free interval and presents within the first two weeks following an operation. The median time of presentation is on post-operative day 6 [2]. Postoperative intussusception is a rare diagnosis. POI is a serious complication in post-operative patients and requires high degree of suspicious for the diagnosis.

\section{CASE HISTORY}

A one and half year old male child was presented to us with the complaint of the lump abdomen for last three months. It was gradually increase in size. On examination it was soft cystic with smooth surface. Routine blood investigations were within normal limits. Ultrasonography (USG) abdomen showed a hypoechoic lesion $(85 \times 90 \mathrm{~mm})$ in size, present in epigastic, left umbilical and hypochondrium region, large abdominal cyst. Contrast enhanced computed tomography showed non enhancing homogeneous thin walled large cystic lesion of size $95 \times 100 \times 92 \mathrm{~mm}$ seen in left lumbar region. It was closely abutting pancreas. Fat plane with surrounding structures were maintained, suggestive of mesenteric cyst (Fig. 1). We planned for exploration; there was a large cyst in the abdomen displacing the bowel with adherent to stomach and the pancreas. Complete excision of the cyst was done. Histopathological showed pseudo cyst of the pancreas. Post-operative period was uneventful till 5th day. Patient initially passed motion, thereafter there was gradual distension of the abdomen and not passed motion. There was

*Address correspondence to this author at the Department of Pediatric Surgery

S.M.S. Medical College and Group of Hospitals, Jaipur, Rajasthan, India.

E-mail:dr.adisms@gmail.com no bilious discharge with soft abdominal distension. Patient was treated conservatively till seventh day. We repeated $\mathrm{x}$-ray abdomen several times, it showed multiple air fluid levels. Ultrasonography abdomen showed dilated bowel loops. We explored the patient; there was ileo-ileal intussusception without lead point. We tried to reduce but could not reduce and resection and anastomosis was done (Fig. 2). Post operative period was uneventful with a excellent recovery. Patient was discharged on 7 th post operative day.

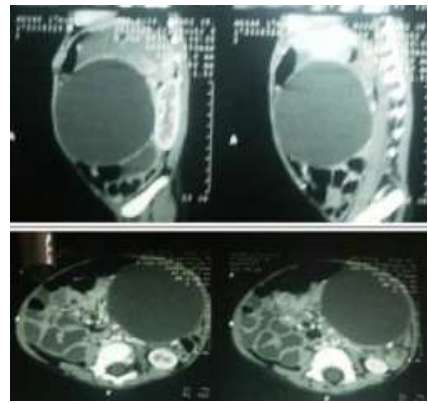

Fig. (1). CECT Image.

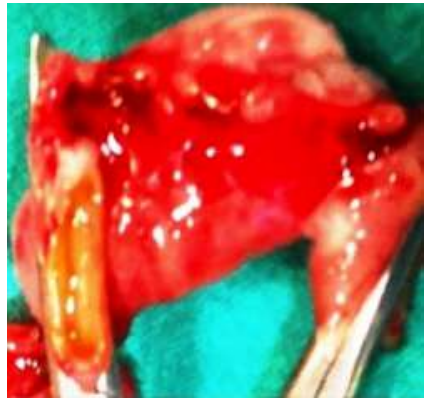

Fig. (2). Excised Specimen of the Intussusception.

\section{DISCUSSION}

Postoperative intussusception is an unusual cause of bowel obstruction after major abdominal operations. It has been reported to occur after $0.08 \%$ to $0.5 \%$ of all major abdominal surgeries. It also causes $5-10 \%$ post operative bowel obstruction in children. In our case it was occurred after excision of the pseudo cyst of pancreas.

The classical signs and symptoms of the intussusception are usually absent in POI. The intussusception usually present with bilious vomiting, intermittent abdominal pain, red currant jelly stools, and an abdominal mass. While in POI signs and symptoms are abdominal distension, bilious vomiting or persistent high nasogastric tube output. Our case had only one episode of the bilious vomiting without bilious

www.njhsciences.com 
nasogastric tube drainage, abdominal distension with constipation. It is slightly more common in male. It is very difficult to distinguish between post operative ileus and POI because both have take days to develop significant abdominal distension. POI should be included in the differential diagnosis of the post operative obstruction in the pediatric age group to prevent fatal complications [3].

POI may be prevented by minimally invasive abdominal approaches [4]. The underlying mechanism of POI is unclear. There are some mechanisms for the POI, which include early postoperative adhesions, prolonged and excessive bowel manipulation, electrolyte disturbances, anesthetic drugs, opioid analgesics, and neurogenic factors [5, 6]. POI is commonly seen before two years of the age.

The clinical evaluation as well as radiologic investigation should also be performed. Erect abdominal X-ray is a primary tool to diagnose and differentiate between obstruction and paralytic ileus. Ultrasonography is useful in POI as in primary intussusception [7], even though it could be more difficult to recognize in the small bowel [8]. Ultrasound and computed tomography are useful investigations [9]. However definite diagnosis may be difficult and can only be made on laparotomy. In our case only x-ray abdomen erect showed multiple air fluid levels while USG abdomen showed dilated bowel loops only.

Most of the authors are not recommend colonic enema for the reduction [4, 9]. POI usually have small bowel intussusception, however, up to $5 \%$ of cases are ileocolic [2]. Small bowel intussusceptions are seen in POI. Colonic enema is not effective in small bowel intussusception and also be hazardous for the bowel anastomosis.

POI does not have any lead point. There are greater risk of intestinal ischemia and necrosis in the patients who are not diagnosed early and a need for operative bowel resection.

\section{CONCLUSION}

POI is a rare surgical complication. There should be keep a differential diagnosis of POI in any case of the postoperative abdominal distension and bilious vomiting. Timely surgical exploration will preserve intestinal viability with excellent outcomes.

\section{CONFLICT OF INTEREST}

Declared None.

\section{ACKNOWLEDGEMENTS}

Declared None.

\section{REFERENCES}

[1] Bai YZ, Chen H, Wang WL. A special type of postoperative intussusception: Ileoileal intussusception after surgical reduction of ileocolic intussusception in infants and children. J Pediatr Surg 2009; 44: 755-8. DOI: 10.1016/j.jpedsurg.2008.08.011

[2] Yang G,Wang X, JiangW, Ma J, Zhao J, LiuW. Post-operative intussusceptions in children and infants: a systematic review. Pediatr Surg Int 2013; 29: 1273-9. DOI: $10.1007 / \mathrm{s} 00383-013-3345-1$

[3] Luckey A, Livingston E, Tache Y. Mechanisms and treatment of postoperative ileus. Arch Surg 2003; 138: 206. DOI: 10.1001/archsurg.138.2.206

[4] Klein JD, Turner CG, Kamran SC, Yu AY, Ferrari L, Zurakowski D. Pediatric postoperative intussusception in the minimally invasive surgery era: A 13 year, single center experience. J Am Coll Surg 2013; 216: 1089-93. DOI: 10.1016/j.jamcollsurg.2013.01.059

[5] Laje P, Stanley CA, Adzick NS. Intussusception after pancreatic surgery in children: A case series. J Pediatr Surg 2010; 45: 1496-99. DOI: 10.1016/j.jpedsurg.2009.09.021

[6] Pumberger W, Pomberger G, Wiesbauer P. Postoperative intussusception: A overlooked complication in pediatric surgical oncology. Med Pediatr Oncol 2002; 38:208-10. DOI: $10.1002 /$ mpo. 1313

[7] Yalcin S, Ciftci AO, Karaagaoglu E. Do radiologic studies correlate with each other and with surgical findings in intussusception? Turk J Pediatr 2008; 50: 336-41.

[8] Emil S, Shaw X, Laberge JM. Post-operative colocolic intussusception. Pediatr Surg Int 2003;19: 220-22.

[9] Wang N, Cui XY, Liu Y, Long J, Xu YH, Guo RX. Adult intussusception: A retrospective review of 41 cases. World $\mathrm{J}$ Gastroenterol 2009; 15: 3303-8. DOI: 10.3748/wjg.15.3303 